The Neutral ISM in Starburst Galaxies

ASP Conference Series, Vol. 320, 2004

S. Aalto, S. Hüttemeister, A. Pedlar

\title{
Age-dating a Starburst: Gemini/CIRPASS Observations of the Core of M83
}

\author{
Stuart D. Ryder \\ Anglo-Australian Observatory, P. O. Box 296, Epping, NSW 1710, \\ Australia; sdr@aaoepp. aao.gov.au \\ Johan H. Knapen \\ Department of Physics, Astronomy and Mathematics, University of \\ Hertfordshire, Hatfield, Herts AL10 9AB, U.K. \\ Lisa M. Mazzuca \\ NASA Goddard Space Flight Center, Code 681, Greenbelt, MD 20771, \\ U.S.A. \\ Robert G. Sharp \& Ian R. Parry \\ Institute of Astronomy, Madingley Road, Cambridge CB3 OHA, U.K.
}

\section{Introduction}

The circumnuclear starburst in NGC 5236 (M 83) has been studied photometrically by Harris et al. (2001) using $H S T /$ WFPC2 images in the broad-band near-UV and optical, as well as narrow-band $\mathrm{H} \alpha$ and $\mathrm{H} \beta$ to derive colors and line equivalent widths for 45 clusters. Despite the excellent spatial resolution of these observations, optical photometric analyses such as this suffer from: (i) patchy (and not easily quantifiable) dust extinction; (ii) the fact that the reddening vector parallels the evolutionary tracks in a two-color diagram; and (iii) selection effects, which tend to exclude the very youngest $(t<5 \mathrm{Myr})$ clusters which have strong emission lines, but only a weak stellar continuum. Additionally, it is not possible to distinguish an instantaneous burst of star formation from a constant star formation rate, on the basis of broad-band colors alone.

To help overcome these drawbacks, we have used the Cambridge Infra-Red Panoramic Survey Spectrograph (CIRPASS; Parry et al. 2000) in Integral Field Unit (IFU) mode on the $8.1 \mathrm{~m}$ Gemini South Telescope to map the equivalent widths of the $\mathrm{Pa} \beta$ line and the $\mathrm{CO}(6,3)$ band across much of the circumnuclear star-forming region in M83. As demonstrated by Ryder, Knapen, \& Takamiya (2001) for M100, the combination of two such diagnostics constrains not just the age, but also the burst duration, for any cluster within the IFU field of view, allowing us to compile an unbiased account of the recent star formation history.

\section{Results}

While a quantitative analysis is still in progress, we can already see evidence for a much more complex star formation history than that found by Harris et 

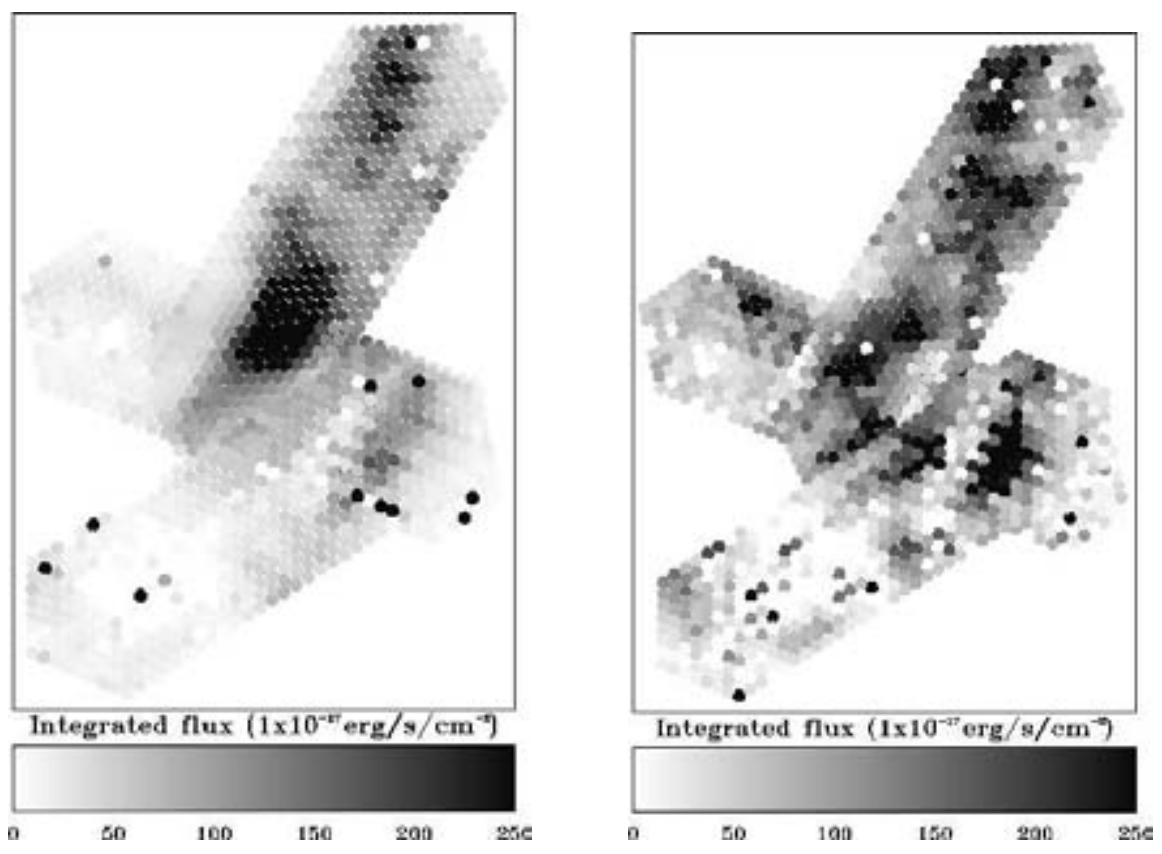

Figure 1. Integrated flux in the $\mathrm{Pa} \beta$ line (left), and in the [Fe II] $1.644 \mu \mathrm{m}$ line (right) from a mosaic of 3 IFU pointings. Each IFU pixel is $0^{\prime \prime} 36$ across, with north at the top, and east to the left. The nucleus of M83 lies near the eastern end of the central IFU position.

al. (2001), or by Thatte, Tecza, \& Genzel (2000) from a single long-slit near-IR spectrum. There is extensive diffuse $\mathrm{Pa} \beta$ emission due west of the nucleus, but only a weak stellar continuum (and thus weak CO absorption), indicative of a very recent burst. Figure 1 compares the distribution of $\mathrm{Pa} \beta$ emission with the [Fe II] $1.644 \mu \mathrm{m}$ emission (which falls within our $H$-band spectral coverage), which is thought to trace shocks due to supernovae within the past $10^{4}$ years (Alonso-Herrero et al. 2003). The [Fe II] is both clumpier and slightly more extended than $\mathrm{Pa} \beta$, suggestive of star formation propagating outwards into regions of previously undisturbed gas.

Acknowledgments. We thank the Raymond and Beverly Sackler Foundation, PPARC, and the Gemini Observatory for their support.

\section{References}

Alonso-Herrero, A., Rieke, G. H., Rieke, M. J., et al. 2003, AJ, 125, 1210

Harris, J., Calzetti, D., Gallagher, J. S., et al. 2001, AJ, 122, 3046

Parry, I. R.; Mackay, C. D.; Johnson, R. A., et al. 2000, SPIE, 4008, 1193

Ryder, S. D., Knapen, J. H., \& Takamiya, M. 2001, MNRAS, 323, 663

Thatte, N., Tecza, M., \& Genzel, R. 2000, A\&A, 364, L47 